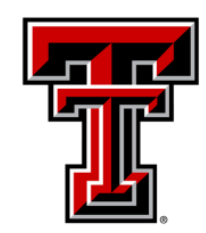

TEXAS TECH UNIVERSITY

Libraries"

\title{
Protective Behavioral Strategies and Alcohol Consumption: Are All Strategies Equal?
}

\section{The Texas Tech community has made this publication openly available. Please share how this access benefits you. Your story matters to us.}

\begin{tabular}{|l|l|}
\hline Citation & $\begin{array}{l}\text { Blanchard, B. E., Stevens, A. K., \& Littlefield, A. K. (2017). Protective } \\
\text { Behavioral Strategies and Alcohol Consumption: Are All Strategies } \\
\text { Equal? Alcoholism Treatment Quarterly, 1-14. } \\
\text { https://doi.org/10.1080/07347324.2017.1387034 }\end{array}$ \\
\hline Citable Link & $\underline{\text { http://hdl.handle.net/2346/73442 }}$ \\
\hline Terms of Use & $\underline{\text { CC-BY }}$ \\
\hline
\end{tabular}


Running head: PROTECTIVE BEHAVIORAL STRATEGIES

Protective Behavioral Strategies and Alcohol Consumption: Are All Strategies Equal?

Brittany E. Blanchard*, MA, Angela K. Stevens, MA, MPH, \& Andrew K. Littlefield, PhD

Texas Tech University 


\begin{abstract}
Given inconsistent findings regarding relations between specific types of alcohol-related protective behavioral strategy use and alcohol outcomes, the current study aimed to examine whether specific strategies vary regarding relations with alcohol consumption. Undergraduate college students $(N=162)$ completed measures online, including the Protective Behavioral Strategy Scale and assessment of alcohol use. A structural equation modeling (SEM) framework was used to examine differential item functioning across alcohol quantity and frequency. Four items demonstrated differential item functioning (e.g., “Avoid drinking games;” “Avoid trying to “keep up” or “out-drink” others”). Findings support idiographic approaches to assessment in treatment.
\end{abstract}

Keywords: protective behavioral strategies; alcohol quantity; alcohol frequency; structural equation modeling; college students 


\section{Introduction}

Given the prevalence of binge drinking among college students (i.e., two-week prevalence of approximately 32\%; Johnston, O’Malley, Bachman, Schulenberg, \& Miech, 2016) and associated negative consequences (e.g., Perkins, 2002; White \& Hingson, 2014), much research attention has been focused on protective behavioral strategies (PBS; e.g., Benton et al., 2004; DeMartini et al., 2013; Madson \& Zeigler-Hill, 2013; Martens, Martin, Littlefield, Murphy, \& Cimini, 2011; Palmer, Corbin, \& Cronce, 2010; Sugarman \& Carey, 2007; see Pearson, 2013 for a critical review). Although definitions of which behaviors constitute PBS vary (e.g., inclusion of abstinence and selective avoidance; Martens et al., 2005; c.f., Sugarman \& Carey, 2007), PBS are typically conceptualized as strategies individuals can use to moderate alcohol consumption and/or reduce associated consequences, consistent with harm-reduction approaches to prevention and treatment (see Marlatt \& Witkiewitz, 2002).

The harm-reduction approach to alcohol intervention provided the foundation for many of the currently used brief alcohol interventions for college students, such as the Brief Alcohol Screening and Intervention for College Students (BASICS; Dimeff, Baer, Kivlahan, \& Marlatt, 1999) and other brief motivational interventions (see Larimer \& Cronce, 2007 for a review). Because many of these interventions include components which aim to increase PBS use, research has begun to evaluate changes in PBS use as a mechanism of action for interventions. However, literature remains largely equivocal, with evidence supporting (e.g., Barnett, Murphy, Colby, \& Monti, 2007; Cimini et al. 2009; Kulesza, McVay, Larimer, \& Copeland, 2013) and refuting (e.g., Martens, Smith, \& Murphy, 2013; Murphy et al., 2012; Walters, Vader, Harris, Field, \& Jouriles, 2009) increased PBS use as the mechanism of change in brief interventions. Some explanations for these discrepancies include examination of PBS as a mechanism of 
change in interventions that did not directly target PBS use (e.g., Walters et al., 2009) and use of different assessments to measure PBS use (e.g., Self-Control Questionnaire [Barnett et al., 2007]; Protective Behavioral Strategies Scale [Cimini et al., 2009; Murphy et al., 2013]). This is potentially problematic, as some assessments include abstinence-related activities, whereas others do not. Moreover, several methodological issues remain regarding the assessment of PBS (see Prince, Carey, \& Maisto, 2013 for a critical review). As such, it may be that examinations of PBS use in clinical research are not adequately capturing behaviors engaged in to avoid specific alcohol-related outcomes. Nevertheless, there is some evidence supporting the notion that use of PBS yields reductions in alcohol consumption and related problems as currently measured, even with suboptimal assessment. Thus, sensitive assessments of PBS use are critical in examining the relation of PBS use with alcohol-related behaviors and necessary for establishing (or refuting) increased PBS use as the mechanism of action in brief interventions.

Despite limitations of PBS assessment, efforts to refine the assessment of PBS have yielded more nuanced approaches (i.e., examining specific types of PBS, rather than providing a sum or mean score of combined strategy use; see Prince et al., 2013). The most widely used measure in clinical and naturalistic alcohol research to date, the Protective Behavioral Strategies Scale (PBSS), assesses two strategy types for reducing consumption, as well as strategies aimed at reducing consequences only, resulting in the following three subscales: Manner of Drinking (MOD; e.g., avoid mixing different types of alcohol), Stopping/Limiting Drinking (SLD; e.g., drink water between drinks), and Serious Harm Reduction (SHR; e.g., use a designated driver and other strategies to avoid consequences, rather than consumption; see Martens et al., 2005, 2007, for more on the empirically-driven scale development of the PBSS). Although some research suggests that all three subscales of the PBSS are negatively associated with alcohol 
consumption and consequences in cross-sectional research (e.g., Martens et al., 2007; Pearson, Kite, \& Henson, 2012), some research suggests more that specific PBS use subscale-alcohol outcome relations may be stronger. For example, increases in MOD use (but not SLD use) have been found to be predictive of decreases in alcohol consumption longitudinally at the betweenand within-person level (e.g., Martens et al., 2011; Napper, Kenney, Lac, Lewis, \& LaBrie, 2014; Pearson, D’Lima, \& Kelley, 2013). Cross-sectionally, MOD use also demonstrated the strongest negative correlation with alcohol consumption quantity (but not frequency) when compared to other PBSS subscales, as well as other PBS measures (i.e., the Protective Behavioral Strategies Measure and the Strategy Questionnaire; Pearson et al., 2012). Martens et al. (2011) found increases in SHR use yielded reductions in negative consequences over one year, though this effect was not replicated when examined at the daily-level for two weeks (i.e., Pearson et al., 2013). Further, SHR use is often found to be negatively associated with alcohol quantity and frequency outcomes in cross-sectional naturalistic research (e.g., Martens et al., 2005, 2007). However, these strategies are to be used when intoxicated (e.g., use a designated driver, know where your drink has been) and, conceptually, should not correlate negatively with consumption (e.g., Pearson et al., 2012). Paradoxically, some PBS strategies have been linked to an increase in alcohol consumption (e.g., Sher, Gotham, \& Watson, 2004; Sugarman \& Carey 2007). For example, Sher et al. (2004) found that, among college students with chronic alcohol use disorders, frequency of pacing behaviors increased over time while in college, indicating that PBS use reflected failed attempts to moderate consumption. Thus, a more nuanced examination of relations between specific PBS and alcohol consumption is warranted.

\section{Current Study}


The PBSS is the most frequently used measure of PBS use in brief alcohol intervention research examining PBS as the mechanism of action (e.g., Barnett et al., 2007; Martens et al., 2013; Murphy et al., 2012). Examining this measure at the item level may help elucidate specific strategies that may be associated with increased or decreased alcohol consumption. Thus, aims of the current study were to examine PBSS items in relation to alcohol consumption using structural equation modeling (SEM) to determine if differential item functioning (i.e., whether the item significantly relates to the outcome after accounting for the relation through the latent variable) was present. Although item-level analyses have previously been conducted on the PBSS (e.g., Bonar et al., 2011; Martens et al., 2007; Treloar, Martens, \& McCarthy, 2014), no work, to our knowledge, has yet to examine differential item functioning of the PBSS for quantity and frequency of alcohol consumption, separately.

We hypothesized that specific items will differentially relate to consumption as a function of how directly the strategy targets the outcome. For example, we predicted that specific SLD strategies (e.g., “Determine not to exceed a certain number of drinks,”) will be related to quantity of average past-month consumption, but not frequency, over and above relations through the latent variable of SLD. Exploratory analyses were conducted on remaining items to determine if any related to consumption over and above relations with the latent construct (i.e., the respective PBSS subscale), as this could indicate sensitive or problematic items on the PBSS.

\section{Methods}

\section{Participants}

Participants $(N=162)$ consisted of undergraduate volunteers in introductory psychology courses from a large southwestern university who completed a battery of demographic questions and self-report measures online. Participants received one research course credit for their 
participation. All procedures and measures were approved by the university’s Institutional Review Board.

\section{Measures}

Demographics. Participants were asked a series of demographic questions, including age, sex, sexual orientation, race, ethnicity, housing situation, and marital status.

Alcohol Consumption. Participants were provided with a National Institute on Alcohol Abuse and Alcoholism (NIAAA) standard drink graphic and were asked the following items using an open text response format: "Think specifically about the past 30 days. During the past 30 days, on how many days did you drink one or more drinks of an alcoholic beverage?” and “On the days that you drank during the past 30 days, how many drinks did you usually have each day? Count a drink as a can or bottle of beer, a wine cooler or glass of wine, champagne, or sherry, a shot of liquor or a mixed drink of cocktail.” Participants were then referred again to the graphic for reference of what constitutes a standard drink.

Protective Behavioral Strategies Scale (PBSS). The 15-item PBSS was used to measure PBS use “when using alcohol or partying” (Martens et al., 2005, p. 702) on a 6-point Likert-like scale, ranging from (1) Never to (6) Always. Coefficient alpha estimates for each subscale ranged from acceptable-to-excellent: Stopping/Limiting Drinking (SLD) $\alpha=$.92, Manner of Drinking (MOD) $\alpha=.86$, and Serious Harm Reduction (SHR) $\alpha=.79$.

\section{Analytic Approach}

Because the PBSS uses 6-point Likert-type response options, these indicators were treated as categorical in all analyses (i.e., a weighted least squares mean and variance [WLSMV] estimator was used, as recommended for ordinal indicators [Muthén \& Muthén, 2012]). First, distributions of the response options of the PBSS items were analyzed. Distributions of the item 
responses for the PBSS indicated low endorsement frequencies for several response options across multiple items. Items were then rescaled such that low (i.e., less than $10 \%$ of the total sample $[n<16])$ endorsement categories were collapsed (e.g., Never/Rarely, Occasionally/Sometimes, Usually/Always), which resulted in nine rescaled items with responses options ranging from 4-5 categories. Participants with missing data on relevant covariates and outcomes for were excluded for specific analyses $(n=3-5)$. Data management and coding was conducted with SAS 9.4 ${ }^{\mathrm{TM}}$ software (SAS Institute Inc., Cary, NC, USA), ${ }^{1}$ and structural equations were modeled using MPlus 7.2 software (Muthén \& Muthén, 1998-2012).

Next, a confirmatory factor analysis of the original three-factor model was tested. Item 9 (i.e., drink shots of liquor, the only reverse-scored item) was removed (e.g., Madson et al., 2013; Treloar et al., 2015). A series of multiple-indicators multiple causes (MIMIC) models were used to examine differential item functioning of each PBSS item. Specifically, average drinking day quantity and past-month number of days drinking were modeled as continuous, independent covariates predicting all three PBSS latent variables. Each item was then regressed onto the consumption variable, one item at a time. Although use of MIMIC models would allow for examination of the consumption variables simultaneously, we chose to conduct analyses separately to be consistent with analytic approaches often used in clinical research (e.g., Cimini et al., 2009; Martens et al., 2013). Given our modest sample size and the necessity of multiple models for testing differential item functioning, a post-hoc binomial probability was calculated to determine the likelihood of finding the number of significant tests (or more) due to chance as a function of the number of models examined.

\section{Results}

\footnotetext{
${ }^{1}$ Copyright @ [2002-2014] SAS Institute Inc. SAS and all other SAS Institute Inc. product or service names are registered trademarks or trademarks of SAS Institute Inc., Cary, NC, USA.
} 


\section{Descriptive Statistics}

Participants ranged in age from 18-29 $(M$ age $=19.72, S D=1.86)$ and self-identified primarily as female (53\%), White (78\%), non-Hispanic (79\%), and living on campus (63\%). Number of days drank in the past month ranged from $0-25(M=4.76, S D=5.53$, skew $=1.51$, kurtosis $=2.11)$, and average past-month quantity ranged from $0-15(M=3.48, S D=3.22$, skew $=1.01$, kurtosis $=1.14)$. Overall, the PBSS subscales were highly correlated (e.g., $r$ s $=.82-$ .91) and negatively associated with both, quantity and frequency of alcohol consumption. For item- and subscale-level means for the PBSS and standardized estimates from the MIMIC models, see Table 1.

\section{CFA and MIMIC Model}

Some model fit indices suggested good fit (i.e., CFI $=.97)$, whereas others indicated suboptimal fit, $\chi^{2}(91)=4822.09, p<.001$, RMSEA $=.12$, for the three-factor (i.e., SLD, MOD, and SHR) CFA, but this was expected given previous work examining the fit of the original three-factor PBSS (e.g., Cadigan, Weaver, McAfee, Herring, \& Martens, 2015; Pearson et al., 2013). Although RMSEAs are reported for transparency, it is notable this measure is particularly sensitive to sample size (see Chen et al., 2008), and thus we focus on CFI in terms of model fit. Further, good fit appears to be found when items were modeled as continuous, rather than categorical, and error variances of two items were correlated (e.g., Martens et al., 2007) or one item was dropped (e.g., Pearson et al., 2012) to improve model fit. In our study the correlation between MOD and SLD scores was significantly high (i.e., $r=.90$; similar to the findings of Madson et al., 2013). Nevertheless, the three-factor model was retained due to its prevalence in the literature (see Pearson, 2013). When frequency and typical quantity were examined as 
independent covariates of the CFA (i.e., the MIMIC model), similar model fits were observed (i.e., $\chi^{2}(85)=231.40-262.71$, CFI = .95-.96, RMSEA = .11-.12).

\section{Differential Item Functioning}

Attempts to model differential item functioning using the three-factor CFA resulted in significantly worse fit (i.e., CFI .4, RMSEA .4) due to high factor intercorrelations; thus, MIMIC models of individual subscales were used to examine item functioning. CFAs for each subscale were then tested, and model fit was adequate for $\operatorname{MOD}$ (i.e., $\chi^{2}(2)=4.98, p=.08$, CFI $=$ $1.00, \mathrm{RMSEA}=.09)$, and $\operatorname{SLD}\left(\chi^{2}(14)=88.24, p<.01, \mathrm{CFI}=.98\right.$, RMSEA $\left.=.18\right)$. SHR was just- identified, so fit indices were not interpretable.

Next, MIMIC models were tested for each subscale and alcohol outcome, which resulted in very similar model fit across SLD and MOD models. Model fit, as indicated by CFIs, suggested good fit for differential item functioning MIMIC models (i.e., CFIs $=.97-1.00$ ), though RMSEAs (i.e., .00-.21) and chi-square estimates (i.e., $\chi^{2}(1-19)=223.83-266.629$ ) varied with regard to fit. ${ }^{2}$ SHR MIMIC models, however, exhibited excellent fit (i.e., $\chi^{2}(2)=$ $.24-.47$, ps $=.79-.88$, CFIs $=1.00$, RMSEAs $=0.00)$. See Figure 1 for the conceptual model. See Table 1 for item-level means and standard deviations, as well as standardized estimates from differential item functioning analyses. Overall, MIMIC models suggested differential item functioning by frequency and quantity for two items (i.e., "Avoid drinking games” [ $\beta \mathrm{s}=-.26$, $.31, p s<.01, .001]$ and "Avoid trying to "keep up" or "out-drink" others;" [ $\beta \mathrm{s}=.21, .19$, ps < .05), and two items differed as a function of quantity (i.e., "Drink slowly, rather than gulp or chug" $[\beta=19, p<.05]$ and "Determine not to exceed a set number of drinks" $[\beta=-.16, p<.01])$.

\footnotetext{
${ }^{2}$ Because item-level analyses were of interest, we proceeded with differential item functioning despite lack of support for model fit. Moreover, many researchers warn about the overreliance on fit statistics when evaluating research questions (see Tomarken \& Waller, 2003).
} 
The binomial probability of finding 6 or more significant results given an alpha level of .05 and 28 item-level tests run is .002.

\section{Discussion}

This study examined whether items of the Protective Behavioral Strategies Scale differ in magnitude of their relation to specific alcohol consumption outcomes (i.e., past-month frequency and average past-month quantity) using a series of multiple-indicators multiple causes (MIMIC) models. Findings suggested differential item functioning for four items, which partially supported our hypotheses. More specifically, “Avoid drinking games” was negatively associated with past-month frequency and typical quantity above and beyond its association with these outcomes through Manner of Drinking (i.e., compared to those with lower drinking levels, those higher in past-month frequency and typical quantity would be less likely to agree with the item “Avoid drinking games” while holding the level of the manner of drinking [MOD] factor constant), which was not predicted. Further, the MOD items "Drinking slowly, rather than gulp or chug,” and “Avoid trying to "keep up” or “out-drink” others” were significantly positively associated with quantity of use. However, “Determine not to exceed a set number of drinks,” was significantly negatively associated with quantity, but not frequency, of past-month consumption, consistent with our hypotheses.

The current findings of differential item functioning across alcohol consumption outcomes on the PBSS have several research and clinical implications. Overall, our results emphasize the need for researchers to assess strategy-specific alcohol-related outcomes in naturalistic and clinical research (e.g., assessing quantity reductions associated with increases in strategies that are specifically aimed at reducing quantity). Further, measurement invariance across levels of a given outcome (e.g., alcohol quantity) should be established when examining 
psychometric properties of a protective behavioral strategies (PBS) assessment. More broadly, the PBSS subscales were negatively associated with both quantity and frequency of alcohol consumption. The effect sizes for serious harm reduction (SHR) use in relation to alcohol consumption outcomes were lower in magnitude (i.e., small-to-medium) in comparison to stopping/limiting drinking (SLD) and MOD use effects (i.e., medium-to-large). Nevertheless, it is unclear why these strategies would be associated with reduced quantity or frequency, as these behaviors are only meant to avoid consequences associated with alcohol-related vehicular accidents and general safety (e.g., going home with a friend).

It is also difficult to interpret differential item functioning, as the residual variance is, by default, unexplained. Speculatively, it may be that in addition to moderating present consumption directly more than other MOD strategies (e.g., “Avoid mixing different types of alcohol”), students may avoid drinking games by avoiding contexts in which drinking games are more likely (e.g., parties), thus reducing their frequency of drinking. Indeed, previous work suggests that students report lower self-efficacy of PBS use when at a bar or party in comparison to their dorm room/apartment (Bonar et al., 2011). This would suggest that selective avoidance (e.g., “Choose to avoid situations where heavy drinking is likely” on the Strategy Questionnaire; Sugarman \& Carey, 2007) may be in college students’ repertoire of strategies to indirectly control their consumption. Given this, identifying alternative behaviors and contexts which promote abstinence or more moderate drinking (in comparison to bars or parties) may be useful for college students aiming to reduce drinking. Further, research examining whether increased PBS use is a mechanism of action for alcohol interventions should assess PBS using the most encompassing definition (i.e., behaviors engaged in before, during, and after drinking, as well as alternative behaviors which promote abstinence; e.g., Sugarman \& Carey, 2007). 
Setting limits was also negatively associated with quantity beyond its relation through SLD use in the current study. However, qualitative studies have reported student focus groups “discussed difficulties in keeping within a preset drinking limit” (Howard, Griffin, Boekeloo, Lake, \& Bellows, 2007, p. 253). Moreover, male college students indicated that their female counterparts appear to limit themselves to one drinking game, whereas males self-reported difficulties stopping (Howard et al., 2007). Thus, teaching students (particularly males) to limit or avoid drinking games may be effective in helping them moderate their alcohol consumption, especially in contexts in which heavy drinking is likely.

Avoiding “keeping up” with or “out-drinking” others was associated with greater alcohol consumption frequency and quantity, after adjusting for MOD PBS use. "Drink slowly, rather than gulp or chug” was also exhibited positive differential relations with average past-month quantity of consumption. Again, although it is difficult to interpret differential item functioning, speculatively, it is possible that these strategies are course-referenced dynamic predictors of alcohol consumption (e.g., Sher et al., 2004). For example, Sher et al. (2004) found that during freshmen year of college, students who initially met criteria for an alcohol use disorder and later remitted engaged in higher frequencies of pacing and drinking low alcohol beverages, which declined with remission. However, among those who initially met criteria and did not remit, their use of these strategies increased over time, reflecting failed attempts to moderate consumption. Thus, it is possible that, like pacing, avoiding keeping up with others reflects efforts to control problematic consumption. This highlights the importance of examining strategy effectiveness and problem recognition in the context of PBS use and the goal of moderate drinking. For example, it may be that college students who engage in problematic alcohol use recognize alcohol-related negative consequences but continue to use ineffective PBS, such as pacing. 
Therefore, researchers and clinicians may benefit from accounting for the role of current alcohol use status and/or problem recognition when assessing PBS use among college students and verify that assessments are invariant across different levels of alcohol-related outcomes.

\section{Limitations and Future Directions}

Despite the contributions of the current study, notable limitations remain, including use of self-report data. Due to the modest sample size $(N=162)$, it is possible that our analyses were underpowered. Based on Monte Carlo simulations by Wolf, Harrington, Clark, and Miller (2013), the number of factors, high factor intercorrelations, and high factor loadings of the models tested in the current study suggest adequate power, though our analyses may be affected by parameter bias due to sample size. Further, assessing quantity via the National Institute of Alcohol Abuse and Alcoholism (NIAAA, 2004) definition (i.e., five or more standard drinks [four for females] within two hours), rather than average past-month quantity, would have been ideal.

The current sample exhibited homogeneity (i.e., nearly $80 \%$ who identified as White and non-Hispanic) and limited age range. Moreover, previous research indicating that relations between PBS use (as assessed by the PBSS subscales) and alcohol outcomes differ by race (e.g., LaBrie, Lac, Kenney, \& Mirza, 2011; Madson \& Zeigler-Hill, 2013). Therefore, our findings may not generalize to more diverse college populations or non-college attending adults. Moreover, several studies have indicated that gender is related to PBS use, such that women consistently use more PBS compared to their male counterparts (e.g., Nguyen, Walters, Wyatt, \& DeJong, 2011; Pearson, 2013). However, in light of evidence that the PBSS does not exhibit measurement invariance across gender (i.e., latent means [i.e., PBSS subscale scores] cannot be meaningfully compared between groups; Treloar et al, 2014), examining gender differences 
would not have been appropriate. These results are also limited by the cross-sectional design, and longitudinal studies are necessary. Thus, while we acknowledge that our findings may not be replicated in larger, more diverse samples, it is our hope that this work will encourage researchers to replicate these analyses with existing PBSS data, particularly on psychometricallyimproved modified versions of the PBSS (e.g., the PBSS-R [Madson, Arnua, \& Lambert, 2013] and the PBSS-20 [Treloar et al., 2015]), to further refine PBS assessment for clinical and research purposes.

Although alcohol-related consequences were not assessed in the current study, because certain strategies are implemented to reduce alcohol-related consequences only (e.g., "Know where your drink has been at all times”), examining differential item functioning by alcoholrelated consequences analyses are also encouraged. Further, item response theory may be beneficial in discerning which PBS are used most frequently as a function of alcohol-related outcomes. Item-level longitudinal analyses may also help shed light on whether commonlyassessed items represent course-referenced predictors. Clinically, idiographic approaches to PBS use may be optimal when establishing tools for moderation, tracking progress, and testing increased PBS use as the mechanism of action in brief interventions (see Prince et al., 2013 for a discussion on the potential benefits of an idiographic approach to PBS assessment).

\section{Conclusion}

The current study utilized MIMIC models and found differential item functioning by specific alcohol consumption outcomes on four items of the most widely used assessment of protective behavioral strategies in the literature to date (i.e., the PBSS). Although replications are necessary, our findings demonstrated that specific PBSS items are not invariant across quantity and frequency of alcohol use, two primary outcomes of interest in intervention research. 
Moreover, these items are also included in revised versions of the PBSS (i.e., the PBSS-20;

Treloar et al., 2016), which is potentially problematic. In sum, assessments of protective behavioral strategies should be assessed for invariance with regard to outcomes of interest (e.g., alcohol consumption and related consequences). In light of these limitations of existing nomothetic PBS assessments, future clinical research examining increased PBS use as the mechanism of action in brief alcohol interventions may benefit from more idiographic approaches.

\section{References}

Barnett, N. P., Murphy, J. G., Colby, S. M., \& Monti, P. M. (2007). Efficacy of counselor vs. computer-delivered intervention with mandated college students. Addictive Behaviors, 32(11), 2529-2548.

Benton, S. L., Schmidt, J. L., Newton, F. B., Shin, K., Benton, S. A., \& Newton, D. W. (2004). 
College student protective strategies and drinking consequences. Journal of Studies on Alcohol, 65(1), 115-121.

Bonar, E. E., Rosenberg, H., Hoffmann, E., Kraus, S. W., Kryszak, E., Young, K. M., ... \& Bannon, E. E. (2011). Measuring university students' self-efficacy to use drinking selfcontrol strategies. Psychology of Addictive Behaviors, 25(1), 155-161.

Borden, L. A., Martens, M. P., McBride, M. A., Sheline, K. T., Bloch, K. K., \& Dude, K. (2011). The role of college students' use of protective behavioral strategies in the relation between binge drinking and alcohol-related problems. Psychology of Addictive Behaviors, 25(2), 346-351.

Cadigan, J. M., Weaver, C. C., McAfee, N. W., Herring, T. E., \& Martens, M. P. (2015). A confirmatory factor analysis of the Protective Behavioral Strategies Scale among OEF/OIF veterans. Addictive Behaviors, 51, 127-130.

Cimini, M. D., Martens, M. P., Larimer, M. E., Kilmer, J. R., Neighbors, C., \& Monserrat, J. M. (2009). Assessing the effectiveness of peer-facilitated interventions addressing high-risk drinking among judicially mandated college students. Journal of Studies on Alcohol and Drugs, Supplement, (s16), 57-66.

DeMartini, K. S., Palmer, R. S., Leeman, R. F., Corbin, W. R., Toll, B. A., Fucito, L. M., \& O’Malley, S. S. (2013). Drinking less and drinking smarter: Direct and indirect protective behavioral strategies in young adults. Psychology of Addictive Behaviors, 27(3), 615-626.

Dimeff, L. A., Baer, J. S., Kivlahan, D. R., Marlatt, G. A. (Eds.). (1999). Brief alcohol screening and intervention for college students (BASICS): A harm reduction approach. New York: Guilford Press. 
Howard, D. E., Griffin, M., Boekeloo, B., Lake, K., \& Bellows, D. (2007). Staying safe while consuming alcohol: a qualitative study of the protective strategies and informational needs of college freshmen. Journal of American College Health, 56(3), 247-254.

Johnston, L. D., O’Malley, P. M., Bachman, J. G., Schulenberg, J. E. \& Miech, R. A. (2016). Monitoring the Future national survey results on drug use, 1975- 2015: Volume 2, College students and adults ages 19-55. Ann Arbor: Institute for Social Research, The University of Michigan. Available at http://monitoringthefuture.org/pubs.html\# $\underline{\text { monographs }}$

Kulesza, M., McVay, M. A., Larimer, M. E., \& Copeland, A. L. (2013). A randomized clinical trial comparing the efficacy of two active conditions of a brief intervention for heavy college drinkers. Addictive Behaviors, 38(4), 2094-2101.

LaBrie, J. W., Lac, A., Kenney, S. R., \& Mirza, T. (2011). Protective behavioral strategies mediate the effect of drinking motives on alcohol use among heavy drinking college students: Gender and race differences. Addictive Behaviors, 36(4), 354-361.

Larimer, M. E., \& Cronce, J. M. (2007). Identification, prevention, and treatment revisited: Individual-focused college drinking prevention strategies 1999-2006. Addictive Behaviors, 32(11), 2439-2468.

Madson, M. B., Arnau, R. C., \& Lambert, S. J. (2013). Development and psychometric evaluation of the revised Protective Behavioral Strategies Scale. Psychological Assessment, 25, 556-567.

Madson, M. B., \& Zeigler-Hill, V. (2013). Protective behavioral strategies, alcohol consumption, and negative alcohol-related consequences: Do race and gender moderate these associations? Journal of Ethnicity and Substance Abuse, 12(3), 242-258. 
Marlatt, G. A., \& Witkiewitz, K. (2002). Harm reduction approaches to alcohol use: Health promotion, prevention and treatment. Addictive Behaviors, 27(6), 867-886.

Martens, M. P., Ferrier, A. G., Sheehy, M. J., Corbett, K., Anderson, D. A., \& Simmons, A. (2005). Development of the Protective Behavioral Strategies Survey. Journal of Studies on Alcohol, 66(5), 698-705.

Martens, M. P., Martin, J. L., Littlefield, A. K., Murphy, J. G., \& Cimini, M. D. (2011). Changes in protective behavioral strategies and alcohol use among college students. Drug and Alcohol Dependence, 118(2), 504-507.

Martens, M. P., Pedersen, E. R., LaBrie, J. W., Ferrier, A. G., \& Cimini, M. D. (2007). Measuring alcohol-related protective behavioral strategies among college students: further examination of the Protective Behavioral Strategies Scale. Psychology of Addictive Behaviors, 21(3), 307-315.

Martens, M. P., Smith, A. E., \& Murphy, J. G. (2013). The efficacy of single-component brief motivational interventions among at-risk college drinkers. Journal of Consulting and Clinical Psychology, 81(4), 691-701.

Muthén, L. K., \& Muthén, B. O. (1998-2012). Mplus user's guide (7 $7^{\text {th }}$ ed.). Los Angeles, CA: Muthén \& Muthén.

Napper, L. E., Kenney, S. R., Lac, A., Lewis, L. J., \& LaBrie, J. W. (2014). A cross-lagged panel model examining protective behavioral strategies: Are types of strategies differentially related to alcohol use and consequences? Addictive Behaviors, 39(2), 480-486.

National Institute on Alcohol Abuse and Alcoholism. (2004). Council approve definition of binge drinking. National Institute of Alcohol Abuse and Alcoholism Newsletter, Winter 2004(3), 3. 
Nguyen, N., Walters, S. T., Wyatt, T. M., \& DeJong, W. (2011). Use and correlates of protective drinking behaviors during the transition to college: Analysis of a national sample. Addictive Behaviors, 36(10), 1008-1014.

Palmer, R. S., Corbin, W. R., \& Cronce, J. M. (2010). Protective strategies: A mediator of risk associated with age of drinking onset. Addictive Behaviors, 35(5), 486-491.

Pearson, M. R. (2013). Use of alcohol protective behavioral strategies among college students: A critical review. Clinical Psychology Review, 33, 1025-1040.

Pearson, M. R., D’Lima, G. M., \& Kelley, M. L. (2013). Daily use of protective behavioral strategies and alcohol-related outcomes among college students. Psychology of Addictive Behaviors, 27(3), 826-831.

Pearson, M. R., Kite, B. A., \& Henson, J. M. (2012). The assessment of protective behavioral strategies: Comparing prediction and factor structures across measures. Psychology of Addictive Behaviors, 26(3), 573-584.

Perkins, H. W. (2002). Surveying the damage: a review of research on consequences of alcohol misuse in college populations. Journal of Studies on Alcohol, supplement, (14), 91-100.

Prince, M. A., Carey, K. B., \& Maisto, S. A. (2013). Protective behavioral strategies for reducing alcohol involvement: A review of the methodological issues. Addictive Behaviors, 38(7), 2343-2351.

Sugarman, D. E., \& Carey, K. B. (2007). The relationship between drinking control strategies and college student alcohol use. Psychology of Addictive Behaviors, 21(3), 338-345.

Tomarken, A. J., \& Waller, N. G. (2003). Potential problems with" well fitting" models. Journal of Abnormal Psychology, 112(4), 578-598. 
Treloar, H. R., Martens, M. P., \& McCarthy, D. M. (2014). Testing measurement invariance of the Protective Behavioral Strategies Scale in college men and women. Psychological Assessment, 26(1), 307-313.

Treloar, H., Martens, M. P., \& McCarthy, D. M. (2015). The Protective Behavioral Strategies Scale-20: Improved content validity of the serious harm reduction subscale. Psychological Assessment, 27(1), 340-346.

Walters, S. T., Vader, A. M., Harris, T. R., Field, C. A., \& Jouriles, E. N. (2009). Dismantling motivational interviewing and feedback for college drinkers: a randomized clinical trial. Journal of Consulting and Clinical Psychology, 77(1), 64-73.

Wasserstein, R. L., \& Lazar, N. A. (2016). The ASA's statement on p-values: context, process, and purpose. The American Statistician, 70(2), 129-133.

White, A., \& Hingson, R. (2014). The burden of alcohol use: Excessive alcohol consumption and related consequences among college students. Alcohol Research: Current Reviews, 35(2), 201-218.

Wolf, E. J., Harrington, K. M., Clark, S. L., \& Miller, M. W. (2013). Sample size requirements for structural equation models an evaluation of power, bias, and solution propriety. Educational and Psychological Measurement, 73(6), 913-934. 